\title{
Justificación multicriterio y multiatributos de inversiones en robots \\ Un caso de estudio
}

\author{
Jorge Luis García Alcaraz* \\ Jaime Sánchez Leal** \\ Salvador Noriega Morales*** \\ Juan José Díaz Núñez $* * * *$
}

\section{Resumen}

En este artículo se presenta un caso de estudio de evaluación y justificación de un robot industrial, en el que se aplica la técnica denominada TOPSIS (del inglés Technique for Order Preference by Similarity to the Ideal Solution), que integra atributos cuantitativos y cualitativos. Los atributos cualitativos analizados fueron costo, capacidad de carga y velocidad; los atributos cualitativos, la calidad del servicio, facilidad de programación e integración. Se contó con la ayuda de un grupo de decisión integrado por directivos de la empresa que adquirió el robot, quienes determinaron las necesidades que se tenían y establecieron los atributos de evaluación de las alternativas. En la técnica TOPSIS, las alternativas representan vectores en el espacio euclidiano, eligiéndose aquella que tiene la menor distancia euclidiana a una alternativa ideal y la mayor distancia euclidiana a una alternativa anti-ideal. El caso reportado se llevó a cabo en una empresa del ramo automotriz ubicada en Santiago de Querétaro en abril del 2005; a trece meses de realizada la adquisición, la técnica y metodología seguida son calificadas como eficientes por el grupo de decisión que las empleó.

Palabras clave: TOPSIS, inversión tecnológica, evaluación de tecnología, selección de alternativas.

Fecha de recepción: 16-08-2005

Fecha de aceptación: 11-01-2007

Multiattribute and Multicriteria Justification for Robot's Investment: A Case of Study

\footnotetext{
* Profesor del Instituto Tecnológico de Celaya. Correo electrónico: jlgarcia@itcj.edu.mx ** Profesor del Instituto Tecnológico de Cd. Juárez. Correo electrónico: jsanchez@itcj.edu.mx

*** Profesor de la Universidad Autónoma de Cd. Juárez. Correo electrónico: snoriega@uacj.mx **** Profesor de la Universidad Autónoma de Cd. Juárez. Correo electrónico: jjdiaz@uacj.mx
} 


\begin{abstract}
In this article appears a case of study in the evaluation and justification of an industrial robot, where the technique denominated TOPSIS is applied (Technique for Order Preference by Similarity to the Ideal Solution), which integrate quantitative and qualitative attributes. The analyzed qualitative attributes were cost, load capacity and speed; the qualitative attributes were the quality of the service, facility for programming and integration capacity. In this study, a decision group integrated by directors of the company that acquired the robot, aid to determined the necessities that were had and settled down the attributes of evaluation for the alternatives. In TOPSIS, the alternatives represent vectors in a Euclidian space, choosing one that has the smaller Euclidian distance to an ideal alternative and the greater Euclidian distance to an anti-ideal alternative. The reported case was carried out in an automotive company located in Santiago de Querétaro, Querétaro, Mexico in April 2005 and the technique and followed methodology are described like efficient by the decision group that used them.
\end{abstract}

Keywords: TOPSIS, technology investment, technology evaluation, selection of alternatives.

\title{
INTRODUCCIÓN
}

$\mathrm{E}^{\mathrm{n}}$ n la actualidad, los estándares de calidad, servicio y costo son muy elevados en los productos industriales, por lo que estas características son objetivos estratégicos de las empresas, que responden invirtiendo en tecnología para la manufactura avanzada (TMA) con el fin de alcanzarlos y mantenerse en el ambiente dinámico de mercado de estos tiempos de globalización, según Lefley et al. (2004). Sin embargo, para Small y Chen (1997), los directivos de las industrias que deciden invertir en TMA se enfrentan a otro tipo de problemas en la selección de la tecnología idónea, ya que existen muchas alternativas de solución en el mercado, atributos involucrados y técnicas de evaluación, lo cual ha generado confusión en los industriales e inversionistas, quienes no tienen confianza en las técnicas de justificación actuales, según Chan et al. (2001).

En lo referente al caso específico de los atributos de evaluación para inversiones en TMA, es ampliamente aceptado que existen dos tipos: los primeros se denominan atributos objetivos, que son medidos generalmente en términos numéricos y representan características de costos e ingeniería, según Braglia y Gabbrielli (2000); mientras que los segundos son denominados subjetivos y para la determinación de éstos se requiere de juicios de personas expertas en el área, quienes basados en su experiencia valoran la contribución de las alternativas con respecto a los atributos en evaluación. Por ejemplo, Parkan y Wu (1998) solicitaron a personas expertas que emitieran su juicio sobre la presencia de éstos en las alternativas en una escala comprendida entre un valor máximo y un valor mínimo; finalmente, los valores obtenidos por cada atributo en cada alternativa fueron promediados. 
Con respecto a las técnicas de evaluación que existen, Chan et al. (2001) las dividen en económicas, estratégicas y analíticas. Las técnicas económicas representan la práctica industrial, sin embargo son ampliamente criticadas por no integrar aspectos cualitativos en el análisis y sus modelos son calificados como ineficientes porque no representan la totalidad del problema de inversión; por su parte, Yusuff et al. (2001) declaran que las decisiones tomadas con estas técnicas conducen generalmente al fracaso de la inversión por ser insuficientes e ignorar atributos cualitativos; para Chan et al. (1999), algunos ejemplos de estas técnicas son el valor presente neto (VPN), tasa interna de retorno (TIR) y costo anual uniforme equivalente (CAUE). Con respecto a las técnicas estratégicas, éstas se basan en los objetivos y misión de la empresa; sin embargo, son criticadas por no integrar aspectos económicos en la evaluación, según Dessureault y Scoble (2000). Finalmente, las técnicas analíticas son poco conocidas, pero tienen la ventaja de poder integrar en la evaluación aspectos económicos, estratégicos, sociales y tecnológicos, por lo que se recomienda ampliamente su uso; algunas de estas técnicas son el proceso de jerarquía analítica (AHP, Analytic Hierarchy Process, por sus siglas en inglés), análisis dimensional (AD), TOPSIS y el modelo lineal aditivo (MLA).

Las técnicas anteriores, multicriterio y multiatributos, aplicadas en la evaluación de TMA aplicada a la industria es ampliamente reportada en la bibliografía especializada; por ejemplo, Boubekri et al. (1991) desarrollaron un sistema experto para la selección y evaluación; Offodile et al. (1987) y Wei et al. (1992) emplearon métodos auxiliados por computadora; Imany y Schlesinger (1989) han propuesto una técnica de programación por metas, la cual ofrecía como resultado una solución semejante a una solución ideal establecida; Knott y Gretto (1982) propusieron, inicialmente, una metodología económica que incorporaba varios costos.

Por lo anteriormente expuesto, se concluye que las inversiones en TMA son un problema complejo, no definido o estructurado, y los enfoques tradicionales (cualitativos, generalmente) consideran en el análisis solamente aspectos operativos y económicos. Además, en Santiago de Querétaro, la manufactura es una de las principales actividades económicas y la inversión en TMA es muy frecuente; aunado a ello los gerentes de empresas se enfrentan a la carencia de técnicas, modelos y metodologías confiables para la evaluación de su tecnología. Por ello, en este artículo se presenta una evaluación multicriterio y multiatributos, que está basado en la técnica TOPSIS (Technique for Order Preference by Similarity to Ideal Solution) desarrollada por Yoon (1980) y que ha sido empleado por los autores en la adquisición de un robot industrial, en el que se evalúan atributos cuantitativos y cualitativos.

\section{METODOLOGÍA}

En esta sección se presenta la metodología seguida para la selección del robot, la cual se dividió en dos fases y está basada en la propuesta de Erol y Ferrel (2004); la primera se 
denomina fase de información, en la que se colectó la información necesaria para el análisis y en la segunda, llamada fase de evaluación, se realizan las operaciones para determinar la alternativa que satisface los atributos evaluados. Un diagrama de las actividades realizadas en cada fase se indica en la figura 1 y se exponen en los siguientes párrafos.

\section{Fase de información}

En esta fase se colecta la información requerida para realizar el análisis y tomar la decisión; estuvo dividida en seis actividades principales que se exponen a continuación.

\section{Identificación de una necesidad u oportunidad}

Esta actividad es producto de la observación de problemas en los sistemas de producción que se tienen instalados en la empresa, los índices tecnológicos y las comparaciones con los competidores. Cuando los directivos consideran que se encuentran en desventaja competitiva, es frecuente que en reuniones de consejo se incorporen espacios en que se discutan las soluciones a este tipo de problemas; generalmente, estas soluciones son inversiones en algún tipo de TMA.

El ingeniero de producción (IP) fue el responsable de identificar las necesidades que tenía su sistema de producción y comunicarlo a los directivos. El problema consistía en mover pequeños lingotes de hierro caliente, los cuales salían de un proceso de corte en una banda y colocarlo en carros transportadores. Esta actividad se realizaba con otra tecnología, pero en meses recientes se han tenido accidentes, que se deseaban eliminar; para ello el Consejo Administrativo ordenó la adquisición de un robot automático.

\section{Integración del grupo de decisión}

El IP fue el responsable de invitar a miembros de diferentes departamentos de la empresa con la finalidad de integrar un grupo de decisión multicriterio. Los integrantes del grupo provenían de los departamentos de Producción, Gerencia General, Mercadotecnia, Investigación y Desarrollo y Finanzas, los que sumaban un total de cinco elementos, todos con derecho de voz y voto. El objetivo de realizar el análisis del problema de inversión mediante un grupo de decisión fue el de integrar los diferentes objetivos y atributos de evaluación y experiencias de los departamentos participantes. 
Figura 1

Metodología de Trabajo

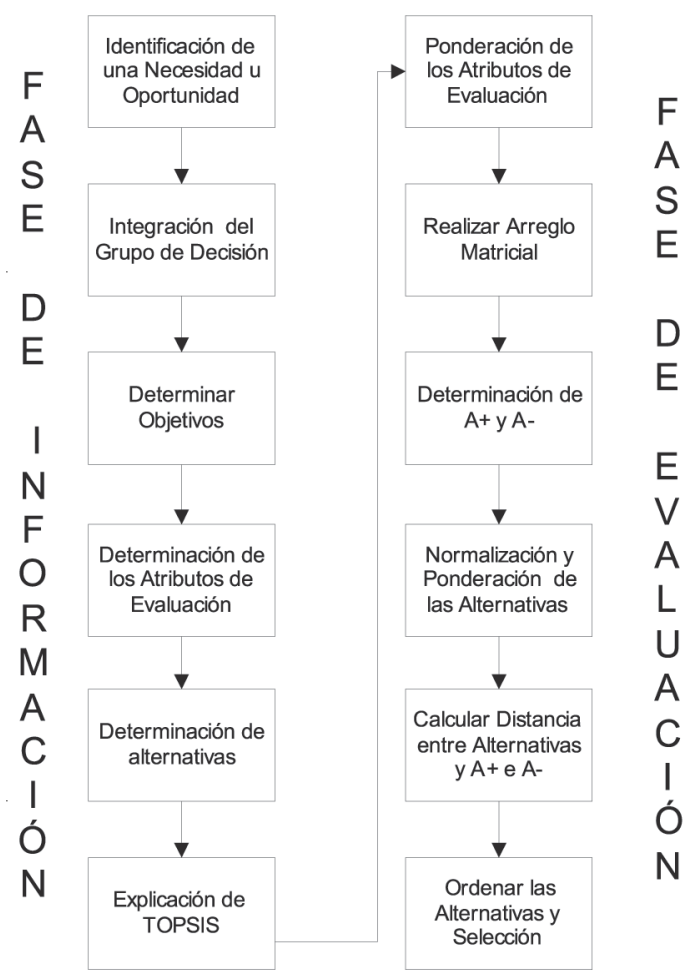

\section{Determinar los objetivos}

Una vez que estaba formado el grupo de decisión, se procedió a establecer los objetivos de la inversión, en la que los diferentes integrantes manifestaron la respuesta o impactos que esperaban por la implantación de la TMA y la fecha estimada para lograrlo. Esto sirvió de base para generar un plan de trabajo mediante el cual se mediría la eficacia del robot en el sistema de producción ya existente y el éxito o fracaso de la inversión. Asimismo, se colectaron opiniones de otros departamentos en los que se esperaba que la TMA tuviera algún impacto.

\section{Estimación de los atributos de evaluación}

En esta actividad cada uno de los integrantes del grupo manifestó cuales serían los atributos de evaluación desde su punto de vista y departamento de trabajo. Para la determinación de los atributos de evaluación se realizó una lluvia de ideas; al conocer las necesidades que la empresa tenía y los objetivos que se deseaban lograr, se determinó que los siguientes atributos deberían ser analizados, a los cuales se les asigna una representación simbólica que se usa a lo largo de todo este trabajo. 
Costo $\left(X_{1}\right)$. Representa el desembolso inicial que debe realizar la empresa para adquirir el robot, los impuestos pagados, costos de mantenimiento preventivo y reparaciones no planeadas. Este atributo está representado en dólares (US\$) y se desea minimizarlo.

Capacidad de carga $\left(X_{2}\right)$. Representa el tonelaje que el robot puede levantar en condiciones normales y extremas. Este atributo es representado en kilogramos $(\mathrm{Kg})$ y se busca maximizarlo.

Velocidad $\left(X_{3}\right)$. Se refiere a la velocidad a la cual el robot mueve los lingotes de la banda transportadora a los carros de transporte. Este atributo, que se busca maximizar, se analiza para saber si las alternativas evaluadas tienen la capacidad de abastecer la velocidad de la banda y se expresa en metros por segundo $(\mathrm{m} / \mathrm{s})$.

Calidad de servicio $\left(X_{4}\right)$. Se refiere a las características del servicio que tiene el proveedor del robot, como son el financiamiento, tiempo de entrega, la capacitación en el manejo del mismo y otros. Este atributo es cualitativo, se basa en los juicios del grupo de decisión y se busca maximizarlo.

Facilidad de programación $\left(X_{5}\right)$. Se refiere a la facilidad con que se puede programar el robot al introducir un nuevo producto con otra forma geométrica diferente, al introducir nuevas coordenadas de posicionamiento, etc. Este atributo es cualitativo, se basa en los juicios del grupo de decisión y se busca maximizarlo.

Integración $\left(X_{\sigma}\right)$. Este atributo se refiere a la facilidad con que el robot se integra al sistema de producción ya existente y con que puede desempeñar otras tareas en otras líneas. Este atributo es subjetivo, se basa en los juicios del grupo de decisión y se busca maximizarlo.

\section{Determinación de las alternativas}

Una vez que se conocen los atributos de evaluación, el departamento de compras proporcionó información sobre posibles alternativas de solución al problema. Después de analizar la cartera de ofertas que se tenían, sus características y los objetivos que se perseguían, se seleccionaron seis robots para su evaluación. En lo sucesivo se denotan por $\mathbf{A}_{1}, \mathbf{A}_{2}, \mathbf{A}_{3}, \mathbf{A}_{4}, \mathbf{A}_{5} \mathbf{y} \mathbf{A}_{6}$.

\section{Explicación de la técnica TOPSIS}

Existen muchos métodos de evaluación multicriterio, algunos de los cuales han sido revisados ampliamente por Hwang y Yoon (1981), Chen y Wang (1992) y Yoon y Wang (1995). Yoon (1980) desarrolló una técnica denominada TOPSIS usando el principio intuitivo de que la alternativa seleccionada deberá tener la menor distancia a una alternativa ideal y a la vez una mayor distancia a una alternativa anti-ideal, según Hwang y Yoon (1981). 
En el proceso de selección por medio de TOPSIS, una alternativa $\mathbf{A}^{\mathbf{k}}$ se considera como un vector en el espacio euclidiano $\mathrm{N}$-dimensional, donde $\mathrm{N}$ es el número de atributos que la caracterizan, tal como se indica en (1).

$$
\mathbf{A}^{\mathrm{k}}=\left(\mathrm{x}_{1}{ }^{\mathrm{k}} \ldots \ldots \ldots \mathrm{x}_{\mathrm{N}}{ }^{\mathrm{k}}\right) \text { para } \mathrm{k}=1,2, \ldots \ldots \mathrm{K}
$$

Similarmente, el vector x-ésimo puede ser analizado como un vector en el espacio Kdimensional, donde $\mathrm{K}$ es el número de alternativas evaluadas y está dado por (2).

$$
\mathbf{X}_{\mathbf{n}}=\left(\mathrm{x}_{\mathrm{n}}{ }^{1} \ldots \ldots \ldots \mathrm{x}_{\mathrm{n}}{ }^{\mathrm{k}}\right) \text { para } \mathrm{n}=1,2 \ldots \ldots \mathrm{N}
$$

La técnica TOPSIS parte del supuesto de que existe una alternativa que debe ser mejor o peor a todas las demás. Así, a la alternativa con las mejores características nominales en los atributos se le llama alternativa ideal $\left(\mathbf{A}^{+}\right)$y está dada por:

$$
\mathbf{A}^{+}=\left(\mathrm{x}_{1}^{+}, \mathrm{x}_{2}^{+}, \ldots \ldots \mathrm{x}_{\mathrm{N}}^{+}\right)
$$

De la misma manera, se genera la alternativa anti-ideal (Ā), que está compuesta por todos los valores nominales no deseados en los atributos dentro de las alternativas y es calculada según (4):

$$
\mathbf{A}-=\left(\mathrm{x}_{1}^{-}, \mathrm{x}_{2}^{-}, \ldots . . \mathrm{x}_{\mathrm{n}}^{-}\right)
$$

Se sugiere que un método directo de realizar la evaluación multicriterio para la elección del robot consiste en elegir la alternativa que posea la menor distancia a la alternativa ideal, de esta manera la alternativa elegida sería muy semejante a la solución ideal, según Srinivasan y Shocker (1973). Otra forma de elegir la alternativa sería eligiendo aquella que se encuentre más alejada de la solución anti-ideal, según Zeleny (1974). TOPSIS es una técnica que considera la distancia a la alternativa ideal y la distancia a la alternativa anti-ideal, la cual puede ser resumida de la siguiente manera:

1. Normalizar cada vector $\mathbf{X}_{\mathbf{i}}$ de los atributos que son sujetos a evaluación y conviértalos a $\mathrm{TX}_{\mathbf{i}}$ según la ecuación (5).

$$
T X_{n}=X_{n} / X_{n}=\left(x_{n}^{1} / X_{n}, \ldots \ldots x_{n}^{k} / X_{n}\right)
$$

dónde $X_{n}$ representa la norma euclidiana del vector (magnitud del vector); así, todos los atributos estarán sin dimensión alguna y se podrán realizar comparaciones entre alternativas; $X_{n}$ está dada por la ecuación (6).

$$
X_{n}=\sum_{1}^{n} x_{i}^{2}
$$


Una vez que los vectores de los atributos se han transformado en vectores unitarios al normalizarse, entonces cada una de las valoraciones de las alternativas estará con coordenadas menores a la unidad, así como los vectores que representan a $\mathbf{A}^{+}$y $\mathbf{A}^{-}$. Una forma de normalizar los vectores de las alternativas de manera directa es usando las ecuaciones (7), (8) y (9).

$$
\begin{aligned}
& T A^{k}=\left(t^{k}, \ldots . t_{n}{ }^{k}\right)=\left(x_{1}^{k} / X_{1}, \ldots \ldots x_{n}{ }^{k} / X_{n}\right) \quad \mathrm{k}=1, \ldots \mathrm{K} \\
& T A^{+}=\left(t^{+}, \ldots t_{n}^{+}\right)=\left(x_{1}^{+} / X_{1}, \ldots \ldots x_{n}^{+} / X_{n}\right) \\
& T A^{-}=\left(t^{-}, \ldots . t_{n}{ }^{-}\right)=\left(x_{1}^{-} / X_{1}, \ldots \ldots . x_{n}^{-} / X_{n}\right)
\end{aligned}
$$

2. Calcular según las ecuaciones (10) y (11) las distancias que existen de los puntos representados por cada alternativa con los puntos que representan a la alternativa ideal y a la alternativa anti-ideal.

$$
\begin{aligned}
& \rho\left(A^{k}, A^{+}\right)=w^{*}\left(T A^{k}-T A^{+}\right)=\sum_{n=1}^{N} w_{1} *\left(t_{n}{ }^{k}-t_{n}^{+}\right)^{2} \\
& \rho\left(A^{k}, A^{-}\right)=w^{*}\left(T A^{k}-T A^{-}\right)=\sum_{n=1}^{N} w^{*}\left(t_{n}{ }^{k}-t_{n}{ }^{-}\right)^{2}
\end{aligned}
$$

Donde $\mathbf{w}$ representa la ponderación o nivel de importancia que los elementos del grupo de decisión han proporcionado al atributo de evaluación.

3. Ordenar los puntos que constituyen la solución de cada alternativa en evaluación de acuerdo con la cercanía y lejanía a las soluciones ideal y anti-ideal, los cuales están dados por la ecuación (12). El criterio o regla de selección que se usa en TOPSIS consiste en elegir la alternativa que contenga el menor valor en el índice $\operatorname{RC}\left(\mathbf{A}^{\mathbf{k}}, \mathbf{A}^{+}\right)$.

$$
R C\left(A^{k}, A^{+}\right)=\frac{\rho\left(A^{k}, A^{+}\right)}{\rho\left(A^{k}, A^{+}\right)+\rho\left(A^{k}, A^{-}\right)}
$$

\section{Fase de evaluación}

Esta fase se encuentra dividida en seis actividades principales en las que se analiza la información obtenida en la fase anterior; éstas se exponen a continuación.

\section{Ponderación de los atributos de evaluación}

En el caso aquí presentado, se recomienda emplear la metodología de ponderación propuesta por Goh et al. (1996) y Parkan y Wu (1998), en la cual se solicita al GD que emita sus 
juicios sobre la importancia que tiene para cada uno de ellos los atributos evaluados, que se realizan en una escala Likert con valores comprendidos entre uno y nueve, donde el uno representa una importancia nula y el nueve indica una importancia extrema o esencial. Las calificaciones obtenidas por cada uno de los atributos son promediados, según (13). El peso o ponderación (w) asignada a cada atributo es el promedio de dicho atributo entre la suma total de los promedios, de acuerdo con (14). De esta manera, la suma de las ponderaciones asignadas al conjunto de atributos es igual a la unidad, como lo indica (15).

$$
\begin{array}{ll}
O_{i}=\sum_{R=1}^{P} O_{i R} & \text { Para } \mathrm{R}=1,2, \ldots \mathrm{P} \\
w_{i}=\frac{O i}{\sum_{i=1}^{N} O_{i}} & \text { Para } \mathrm{i}=1,2 \ldots \mathrm{N} \\
\sum_{i=1}^{N} w_{i}=1 &
\end{array}
$$

Donde:

$O_{i R}$ es juicio emitido por el experto R para el atributo i

$O_{i}$ es el promedio de las asignaciones obtenido por el atributo i.

$w_{i}$ es la ponderación para el atributo i

$\mathrm{N}$ es el número total de atributos

$\mathrm{P}$ es el número de expertos que emiten su juicio

En el cuadro 1, se ilustra el proceso de obtención de la ponderación de los atributos evaluados por los expertos, los cuales se representan por $\mathbf{E}_{\mathbf{1}}, \mathbf{E}_{\mathbf{2}} \ldots \mathbf{E}_{\mathbf{5}}$; los valores asignados por los integrantes del GD se promedian y ponderan de acuerdo con (13) y (14), de tal manera que la suma del vector w es la unidad, cumpliéndose la condición (15).

Cuadro 1

Ponderación de los atributos

\begin{tabular}{|c|c|c|c|c|c|c|}
\hline $\begin{array}{c}\text { Variables } \\
\text { Expertos }\end{array}$ & $\mathbf{X}_{\mathbf{1}}$ & $\mathbf{X}_{\mathbf{2}}$ & $\mathbf{X}_{\mathbf{3}}$ & $\mathbf{X}_{\mathbf{4}}$ & $\mathbf{X}_{\mathbf{5}}$ & $\mathbf{X}_{\mathbf{6}}$ \\
\hline $\mathbf{E}_{\mathbf{1}}$ & 8 & 9 & 8 & 7 & 6 & 8 \\
\hline $\mathbf{E}_{\mathbf{2}}$ & 8 & 8 & 7 & 6 & 5 & 9 \\
\hline $\mathbf{E}_{\mathbf{3}}$ & 6 & 8 & 7 & 9 & 5 & 7 \\
\hline $\mathbf{E}_{\mathbf{4}}$ & 7 & 8 & 6 & 7 & 9 & 7 \\
\hline $\mathbf{E}_{\mathbf{5}}$ & 8 & 5 & 6 & 7 & 9 & 7 \\
\hline Promedio & 7.4 & 7.6 & 6.8 & 7.2 & 6.8 & 7.6 \\
\hline $\boldsymbol{w}$ & 0.17051 & 0.17512 & 0.15668 & 0.16590 & 0.15668 & 0.17512 \\
\hline
\end{tabular}




\section{Realizar arreglo matricial}

En esta actividad, se estimaron los valores que posee cada alternativa con respecto a cada uno de los atributos que se evalúan. En la evaluación se integran atributos cuantitativos y cualitativos. Los primeros fueron obtenidos de las características presentadas por los proveedores de los robots; sin embargo, los segundos fueron estimados mediante juicios de los integrantes del GD, los cuales se emitieron en una escala Likert entre el uno y el nueve. Los valores asignados por los cinco expertos se sumaron y se obtuvo una media aritmética, la cual representa su valor. El cuadro 2 ilustra este proceso de obtención de valores.

Cuadro 2

Obtención de valores para atributos cualitativos

\begin{tabular}{|c|c|c|c|c|c|c|c|c|c|c|c|c|c|c|c|c|c|c|}
\hline \multirow{2}{*}{$\begin{array}{l}\text { Expertos } \\
\text { alternativas }\end{array}$} & \multicolumn{3}{|c|}{$\mathbf{E}_{1}$} & \multicolumn{3}{|c|}{$\mathbf{E}_{2}$} & \multicolumn{3}{|c|}{$\mathbf{E}_{3}$} & \multicolumn{3}{|c|}{$\mathbf{E}_{4}$} & \multicolumn{3}{|c|}{$\mathbf{E}_{5}$} & \multicolumn{3}{|c|}{ Promedio } \\
\hline & $\mathrm{X}_{4}$ & $\mathbf{X}_{5}$ & $X_{6}$ & $\mathbf{X}_{4}$ & $\mathbf{X}_{5}$ & $X_{6}$ & $X_{4}$ & $\mathbf{X}_{5}$ & $X_{6}$ & $\mathbf{X}_{4}$ & $X_{5}$ & $X_{6}$ & $X_{4}$ & $\mathbf{X}_{5}$ & $\mathbf{X}_{6}$ & $\mathbf{X}_{\mathbf{4}}$ & $\mathbf{X}_{5}$ & $X_{6}$ \\
\hline $\mathbf{A}_{1}$ & 6 & 9 & 7 & 6 & 6 & 6 & 3 & 7 & 5 & 7 & 6 & 8 & 4 & 7 & 5 & 5.2 & 7 & 6.2 \\
\hline $\mathbf{A}_{2}$ & 4 & 7 & 5 & 5 & 7 & 6 & 6 & 6 & 6 & 5 & 6 & 6 & 7 & 5 & 6 & 5.4 & 6.2 & 5.8 \\
\hline $\mathbf{A}_{3}$ & 6 & 8 & 5 & 3 & 8 & 5 & 4 & 7 & 6 & 5 & 5 & 5 & 3 & 4 & 4 & 4.2 & .4 & 5 \\
\hline $\mathbf{A}$ & 7 & 2 & 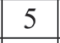 & 7 & 5 & 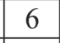 & 6 & - & 6 & 5 & 8 & 8 & 7 & 3 & 8 & 6.4 & 4.8 & 6.6 \\
\hline $\mathbf{A}_{5}$ & 6 & 7 & 7 & 8 & 4 & 7 & 1 & 5 & 7 & 6 & 7 & 7 & 8 & 5 & 6 & 7 & 5.6 & 6.8 \\
\hline $\mathbf{A}_{6}$ & 9 & 6 & 8 & 9 & 3 & 6 & 8 & 4 & 8 & 7 & 6 & 6 & 9 & 6 & 7 & 8.4 & 5 & 7 \\
\hline
\end{tabular}

solución. Estos valores son los que representan a cada alternativa como un vector en el espacio euclidiano y sobre los que se realizan los cálculos; por ejemplo, $\mathbf{A}_{1}$ es representada por $(8500,90,1.4,5.2,7,6.2), \mathbf{A}_{2}$ por $(4750,85,1.3,5.4,6.2,5.8)$ y así sucesivamente.

Cuadro 3

Valores de los atributos y las alternativas

\begin{tabular}{|c|c|c|c|c|c|c|}
\hline $\begin{array}{c}\text { Variables } \\
\text { Alternativas }\end{array}$ & $\mathbf{X}_{\mathbf{1}}$ & $\mathbf{X}_{\mathbf{2}}$ & $\mathbf{X}_{\mathbf{3}}$ & $\mathbf{X}_{\mathbf{4}}$ & $\mathbf{X}_{\mathbf{5}}$ & $\mathbf{X}_{\mathbf{6}}$ \\
\hline $\mathbf{A}_{\mathbf{1}}$ & 8500 & 90 & 1.4 & 5.2 & 7 & 6.2 \\
\hline $\mathbf{A}_{\mathbf{2}}$ & 4750 & 85 & 1.3 & 5.4 & 6.2 & 5.8 \\
\hline $\mathbf{A}_{\mathbf{3}}$ & 6300 & 105 & 0.9 & 4.2 & 6.4 & 5 \\
\hline $\mathbf{A}_{\mathbf{4}}$ & 4800 & 95 & 1.3 & 6.4 & 4.8 & 6.6 \\
\hline $\mathbf{A}_{\mathbf{5}}$ & 7200 & 98 & 1.6 & 7 & 5.6 & 6.8 \\
\hline $\mathbf{A}_{\mathbf{6}}$ & 9400 & 93 & 1.9 & 8.4 & 5 & 7 \\
\hline
\end{tabular}

\section{Determinación de $\boldsymbol{A}^{+} \mathbf{y} \boldsymbol{A}^{-}$}

Los valores de la alternativa ideal $\mathbf{A}^{+}$corresponden a los valores nominales más deseados del cuadro 3 y los valores de $\mathbf{A}^{-}$indican los valores menos deseados. Por ejemplo, el mejor valor del atributo costo es el menor ( $\$ 4750$, de la alternativa $\mathbf{A}_{2}$ ), mientras que el mejor valor del atributo de la velocidad corresponde al más alto $(1.9 \mathrm{~m} / \mathrm{s}$, de la alternativa $\mathbf{A}_{6}$ ); los vectores $\mathbf{A}^{+}$y $\mathbf{A}^{-}$son los que se ilustran en el cuadro 4 . 
Cuadro 4

Alternativas ideal e anti-ideal

\begin{tabular}{|c|c|c|c|c|c|c|}
\hline Alternativas & $\mathbf{X}_{\mathbf{1}}$ & $\mathbf{X}_{\mathbf{2}}$ & $\mathbf{X}_{\mathbf{3}}$ & $\mathbf{X}_{\mathbf{4}}$ & $\mathbf{X}_{\mathbf{5}}$ & $\mathbf{X}_{\mathbf{6}}$ \\
\hline $\mathbf{A}^{+}$ & 4750 & 105 & 1.9 & 8.4 & 7 & 7 \\
\hline $\mathbf{A}^{-}$ & 9400 & 85 & 0.9 & 4.2 & 4.8 & 5 \\
\hline
\end{tabular}

\section{Normalización y ponderación de las alternativas}

Dado que las variables cuantitativas y cualitativas se encuentran expresadas en diferentes unidades de medición, se procede a la normalización de las mismas con la finalidad de que los atributos de evaluación se encuentren en unidades adimensionales y así poder realizar comparaciones entre ellas. Para este cálculo se emplean las ecuaciones 7, 8 y 9. El proceso de ponderación se indica en el cuadro 5.

Cuadro 5

Normalizaciones de los atributos

\begin{tabular}{|c|c|c|c|c|c|c|}
\hline $\begin{array}{c}\text { Variables } \\
\text { Alternativa }\end{array}$ & $\mathbf{X}_{\mathbf{1}}$ & $\mathbf{X}_{\mathbf{2}}$ & $\mathbf{X}_{\mathbf{3}}$ & $\mathbf{X}_{\mathbf{4}}$ & $\mathbf{X}_{\mathbf{5}}$ & $\mathbf{X}_{\mathbf{6}}$ \\
\hline $\mathbf{A}_{\mathbf{1}}$ & 0.49260 & 0.38864 & 0.39886 & 0.33967 & 0.48560 & 0.40369 \\
\hline $\mathbf{A}_{\mathbf{2}}$ & 0.27528 & 0.36705 & 0.37037 & 0.35274 & 0.43010 & 0.37764 \\
\hline $\mathbf{A}_{\mathbf{3}}$ & 0.36511 & 0.45341 & 0.25641 & 0.27435 & 0.44397 & 0.32556 \\
\hline $\mathbf{A}_{\mathbf{4}}$ & 0.27818 & 0.41023 & 0.37037 & 0.41806 & 0.33298 & 0.42973 \\
\hline $\mathbf{A}_{\mathbf{5}}$ & 0.41727 & 0.42319 & 0.45584 & 0.45725 & 0.38848 & 0.44275 \\
\hline $\mathbf{A}_{\mathbf{6}}$ & 0.54476 & 0.40159 & 0.54131 & 0.54870 & 0.34685 & 0.45578 \\
\hline $\mathbf{A}^{+}$ & 0.27528 & 0.45341 & 0.54131 & 0.54870 & 0.48560 & 0.45578 \\
\hline $\mathbf{A}^{-}$ & 0.54476 & 0.36705 & 0.25641 & 0.27435 & 0.33298 & 0.32556 \\
\hline
\end{tabular}

La matriz de valores normalizados del cuadro 5 se multiplica por la ponderación o factor de importancia (w) que tiene cada uno de los atributos de evaluación obtenidos en el cuadro 1. Para la realización de esta operación, se considera al atributo como un vector columna y a la ponderación de ese atributo como un escalar. La matriz que se obtiene se ilustra a continuación en el cuadro 6.

Cuadro 6

Ponderación de atributos normalizados

\begin{tabular}{|c|c|c|c|c|c|c|}
\hline $\begin{array}{c}\text { Atributo } \\
\text { Alternativa }\end{array}$ & $\mathbf{X}_{\mathbf{1}}$ & $\mathbf{X}_{\mathbf{2}}$ & $\mathbf{X}_{\mathbf{3}}$ & $\mathbf{X}_{\mathbf{4}}$ & $\mathbf{X}_{\mathbf{5}}$ & $\mathbf{X}_{\mathbf{6}}$ \\
\hline $\mathbf{A}_{\mathbf{1}}$ & 0.08399 & 0.06806 & 0.06249 & 0.05635 & 0.07608 & 0.07069 \\
\hline $\mathbf{A}_{\mathbf{2}}$ & 0.04694 & 0.06428 & 0.05803 & 0.05852 & 0.06739 & 0.06613 \\
\hline $\mathbf{A}_{\mathbf{3}}$ & 0.06225 & 0.07940 & 0.04018 & 0.04551 & 0.06956 & 0.05701 \\
\hline $\mathbf{A}_{\mathbf{4}}$ & 0.04743 & 0.07184 & 0.05803 & 0.06936 & 0.05217 & 0.07525 \\
\hline $\mathbf{A}_{\mathbf{5}}$ & 0.07115 & 0.07411 & 0.07142 & 0.07586 & 0.06087 & 0.07753 \\
\hline $\mathbf{A}_{\mathbf{6}}$ & 0.09289 & 0.07033 & 0.08481 & 0.09103 & 0.05435 & 0.07981 \\
\hline $\mathbf{A}^{+}$ & 0.04694 & 0.07940 & 0.08481 & 0.09103 & 0.07608 & 0.07981 \\
\hline $\mathbf{A}^{-}$ & 0.09289 & 0.06428 & 0.04018 & 0.04551 & 0.05217 & 0.05701 \\
\hline
\end{tabular}




\section{Calcular distancias entre alternativas}

El concepto de distancias entre las alternativas en evaluación y $\mathbf{A}^{+}$y $\mathbf{A}^{-}$es la base intuitiva de la técnica TOPSIS, ya que se busca la mayor cercanía posible al vector $\mathbf{A}^{+}$, pero a su vez la mayor lejanía a A-. Para el cálculo de esas distancias se emplea las ecuaciones 10 y 11. Los resultados se resumen en el cuadro 7.

Cuadro 7

Distancias entre alternativas

\begin{tabular}{|c|c|c|c|c|}
\hline Alternativa & $\rho\left(A^{k}, A^{+}\right)$ & $\rho\left(A^{k}, A^{-}\right)$ & $R C\left(A^{k}, A^{+}\right)$ & Orden \\
\hline $\mathbf{A}_{\mathbf{1}}$ & 0.05732 & 0.03831 & 0.59937 & 5 \\
\hline $\mathbf{A}_{\mathbf{2}}$ & 0.04760 & 0.05398 & 0.46859 & 4 \\
\hline $\mathbf{A}_{3}$ & 0.06972 & 0.03833 & 0.64524 & 6 \\
\hline $\mathbf{A}_{4}$ & 0.04286 & 0.05782 & 0.42571 & 2 \\
\hline $\mathbf{A}_{\mathbf{5}}$ & 0.03550 & 0.05443 & $\mathbf{0 . 3 9 4 7 4}$ & $\mathbf{1}$ \\
\hline $\mathbf{A}_{\mathbf{6}}$ & 0.05164 & 0.06801 & 0.43156 & 3 \\
\hline
\end{tabular}

\section{Ordenar las alternativas y selección}

En la cuarta columna del cuadro 7 se indica el orden de preferencia que tienen las alternativas analizadas. De acuerdo con el criterio establecido en TOPSIS, la alternativa representada por $\mathbf{A}_{5}$, tiene una relación $\mathbf{R C}$ con un valor de 0.39474 , la cual es menor a las demás alternativas y debe ser elegida. Se observa, además, que esta alternativa mantiene la menor distancia a la alternativa ideal (0.03550), aunque la distancia a la alternativa anti-ideal no es la más lejana (0.05443), ya que en este caso la alternativa $\mathbf{A}_{6}(0.06443)$ es superior a ésta.

Si se usa el criterio de Zeleny en el que se sugiere que se elija la alternativa más cercana a la alternativa ideal $\left(\mathbf{A}^{+}\right)$, entonces la alternativa representada por $\mathbf{A}_{5}$ debe ser elegida; asimismo, si se usa el criterio de elegir la alternativa más lejana a la anti-ideal (A), entonces la alternativa $\mathbf{A}_{6}$ debe ser elegida.

\section{Resultados y Recomendaciones}

Al final de esta evaluación multicriterio se ha logrado aplicar la técnica TOPSIS en la justificación de inversiones en robots y el caso de estudio reportado puede servir de marco de referencia para otras empresas que deseen realizar la misma inversión. En la evolución se integraron atributos cuantitativos y cualitativos en la evaluación de alternativas, mejorando las críticas que se realizan frecuentemente a las técnicas económicas de flujo descontado tradicionales. El robot seleccionado mediante esta técnica no ha presentado problema alguno en su corto periodo de operación y los integrantes del grupo de decisión consideran que fue una buena elección y califican la inversión como eficiente. Finalmente, es conveniente señalar dos aspectos importantes 
que se deben considerar: el primero es que esta metodología responde solamente a las necesidades de esta empresa y que cualquier otra debe realizar su propio análisis de necesidades para determinar los atributos que deben ser analizados y el segundo es que dos grupos de decisión diferentes que evalúan las mismas TMA pueden llegar a concluir que alternativas diferentes deben ser elegidas, aun cuando ambos grupos sean lógicos y racionales, lo cual es debido a la valoración subjetiva de los atributos subjetivos y del proceso de ponderación.

\section{REFERENCIAS}

BOUBEKRI, N., M. SAHOUI y C. LAKRIB, 1991, "Development of an expert system for industrial robot selection”, en Computers and Industrial Engineering, 21, pp. 119-127.

BRAGLIA, M. y R. Gabbrielli, 2000, "Dimensional analysis for investment selection in industrial robots", en International Journal of Production Research, 38(18), pp. 48434848.

CHAN, F. et al., 1999. “An Integrated Approach to Investment Appraisal for Advanced Manufacturing Technology", en Human Factors and Ergonomics in Manufacturing, 9(1), pp. 69- 86.

CHAN, F. et al., 2001, "Investment appraisal techniques for advanced manufacturing technology (AMT): a literature review", en Integrated Manufacturing Systems, 12(1), pp. $35-47$.

CHEN, S. y C. HWANG, 1992, Fuzzy Multiple Attribute Decision Making: Methods and Applications, Springer-Verlag, Berlin, Alemania.

DESSUREAULT, S. y M. SCOBLE, 2000, "Capital investment appraisal for the integration of new technology into mining systems", en Trans. Institution of Mining and Metallurgy, 109, pp. 31-40.

EROL, I. y W. G. Ferrel, 2003, “A methodology to support decision making across the supply chain of an industrial distributor", en International Journal of Production Economics, 89(2), pp. 119-129.

GOH, C., Y. TUNG, y C. CHENG, 1996, “A revised weighted sum decision model for robot selection”, en Computers of Industrial Engineering, 30, pp. 193- 199.

HWANG, C. y K. Yoon, 1981, Multiple Attribute Decision. Making: Methods and Applications, A State of-the-Art Survey, Springer-Verlag, Berlin, Alemania.

IMANY, M. y R. SCHLESINGER, 1989, "Decision models for robot selection: a comparison of ordinary least squares and linear goal programming methods", en Decision Sciences, 20, pp. 40-53. 
KNOTT, K. y R. GRETTO, 1982, “A model for evaluating alternative robot systems under uncertainty", en International Journal of Production Research, 20, pp. 155-165.

LEFLEY, F. et al., 2004. "Manufacturing investments in the Czech Republic: An international comparison", en International Journal of Productions Economics, 88, pp. $1-14$.

OFFODILE, O., B. LAMBERT y R. DUDEK, 1987, “Development of a computer aided robot selection procedure (CARSF)", en International Journal of Production Research, 25, pp. 1109-1121.

PARKAN, C. y L. WU, 1998, "Decision-making and performance measurement models with applications to robot selection", en Computers \& Industrial Engineering, 36(3), pp. 503-523.

SMALL, M. e I. CHEN, 1997, "Economic and strategic justification of AMT inferences from industrial practices", en International Journal of Production Economics, 49(1), pp. 65-75.

SRINIVASAN, V. y A. SHOCKER, 1973, "Estimating the weights for multiple attributes in a composite criterion using pairwise judgments", en Psychometrika, 38, pp. 473493.

WEI, C., A. KAMRANI y H. WEIBE, 1992, "Animated simulation of the robot process capability”, en Computers and Industrial Engineering, 23, pp. 237-240.

YOON, K., 1980, Systems Selection by Multiple Attribute Decision Making, tesis doctoral, Kansas State University Press, Manhattan, Estados Unidos de América.

y C. HWANG, 1995, Multiple Attribute Decision Making: An Introduction, Sage, California, Estados Unidos.

YUSUFF, R., K. P. YEE y M. HASHMIB, 2001, “A preliminary study on the potential use of the analytical hierarchical process (AHP) to predict advanced manufacturing technology (AMT) implementation", en Robotics and Computer-Integrated Manufacturing, 17(5), pp. 421-427.

ZELENY, M., 1974, Linear Multiobjective Programming, Springer-Verlag, Nueva York, Estados Unidos.

A 